\title{
Detection of Seepage Path of dam foundation Based on Groundvue Radar
}

\author{
LU Jianhong, ${ }^{1, a^{*}}$, BAI Yun ${ }^{2, b}$ and WANG $\mathrm{Qi}^{1, \mathrm{c}}$ \\ ${ }^{1}$ College of Earth Science and Engineering, Hohei University, Nanjing, China \\ ${ }^{2}$ Water Conservancy and Hydropower Survey and Design Institute of Qinghai, Xining, China \\ alujianhohai@sina.com, 모slby219715@163.com, wangqihohei@sina.com
}

Keywords: Dam foundation, Groundvue Radar, Seepage path, Detection

Abstract. Location of Nanmenxia Reservoir is very important, and the reservoir has a significant benefit. But the leakage of dam foundation was serious; there was an urgent need to find out leakage location and path. The effect of previous treatment was not obvious, and it is very important to find out the situation of the dam foundation. Through the introduction of British Groundvue series of Ground Penetrating Radar System, the seepage channel was detected, and main leakage paths of dam foundation were looked for. By detecting and analysis, drawed a conclusion that there was obvious strong leakage band in the bedrock, the local obviously broken belt developed on the right bank of the grouting trnnel. It provided technical support for design and construction of danger removal and reinforcement for Nanmenxia Reservoir.

\section{Introduction}

Nanmenxi reservoir was founded in 1974 and put into operation in 1982. It is a medium-sized project mainly used for irrigation. It has complex geological conditions, and leakage is also serious. Nanmenxia reservoir seepage treatment work has already begun as early as 1975 . It has been carried out three stages of curtain grouting, but the effect was not ideal. The dam of Nanmenxia Reservoirs were identified more serious dangerous on the safety appraisal in October 2010. It affected the safety of flood discharge. The reservoir can't normal operation according to the requirements of design. It is a third class dam. To maximize the social and economic benefits of the reservoir and prevent major disasters, the reinforcement of the reservoir will be carried out recently approved by the Ministry of Water Resources.

By introduction of GPR equipment and technology, combined with on-site testing and validation, to confirm Nanmenxia reservoir leakage path and distribution, which is necessary to provide technical support for the reinforcement construction.
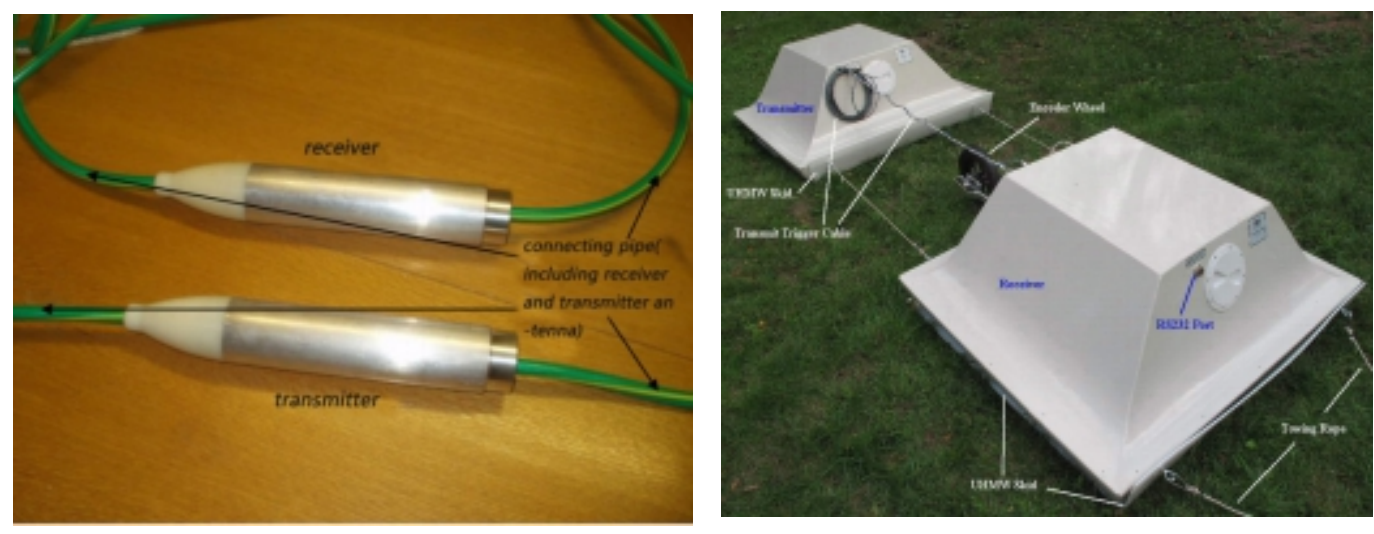

Figure 1 GV series of ground penetrating radar antenna

\section{Groundvue radar system}

Groundvue series of ground penetrating radar test system's main technical indicators: scan rate of four-channel radar host is 250 sweep / sec; $400 \mathrm{MHz}$ antenna frequency range: 200-600 MHz; 50 
MHz Antenna Frequency range: 30-100 MHz; $15 \mathrm{MHz}$ Antenna Frequency range: 5MHz-30 MHz; high pulse repetition rate: $2 \mathrm{M} \mathrm{Hz}$ to $500 \mathrm{KHz}$.

Geological radar antenna according to the classification in different ways can be classified into different types of antennas. From the nature of the work, it can be classified into transmit and receive antennas; from the shield or not shield, it can be classified into shielded and unshielded antenna. as shown in Figure 1.

\section{Indoor test}

For a reasonable analysis of Nanmenxia reservoir's images measured by GV ground penetrating radar, Hohai University established a simulation platform based on $1 \mathrm{GHz}$ of $\mathrm{GV} 3$ ground penetrating radar antenna in the laboratory, to understand the characteristics of the target model image measured by GV ground penetrating radar. As shown in Figure 2.

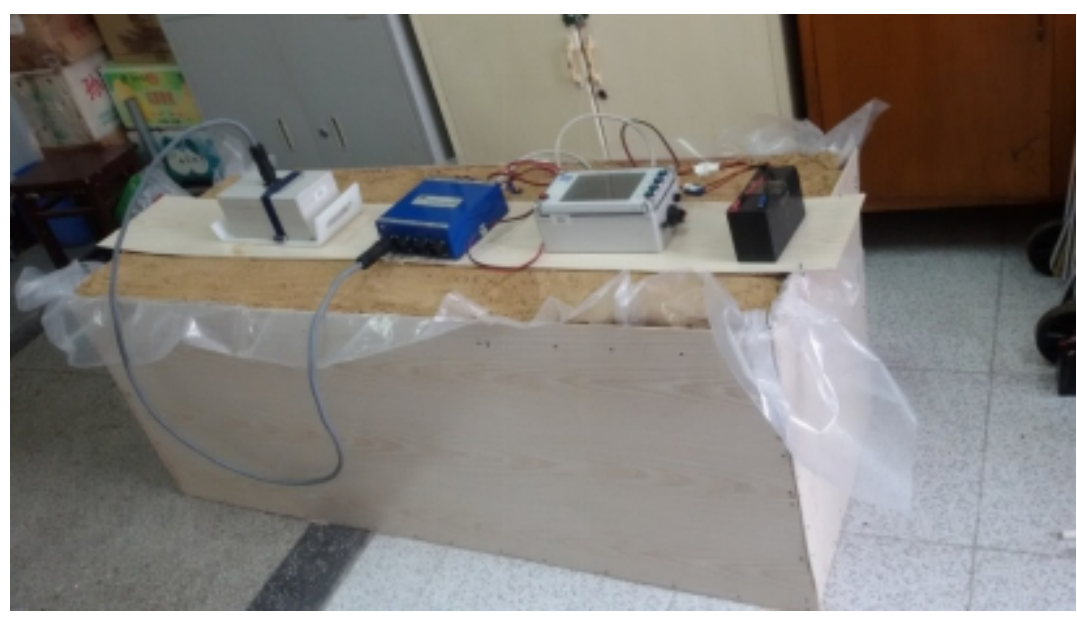

Figure 2 GV ground penetrating radar indoor test platform

\section{Field test}

The main survey line is arranged in the top and fore slope of the dam. The main detecting route include: three equidistant longitudinal survey lines along the highway direction on the dam crest, two longitudinal survey lines on the dam fore slope grouting platform, they were GV2 and GV6 detection; Dam fore slope survey lines were detected from grouting platform to the top of slope. It's direction perpendicular to the dam's trend. Because there are many stone on dam slope, it was not conducive to lager size and weight GV2's probe (smaller effect on the light GV6), so the measuring space was arranged to $3.5 \mathrm{~m}$. The total of survey lines is 26 . Test method is shown in Figure 3.
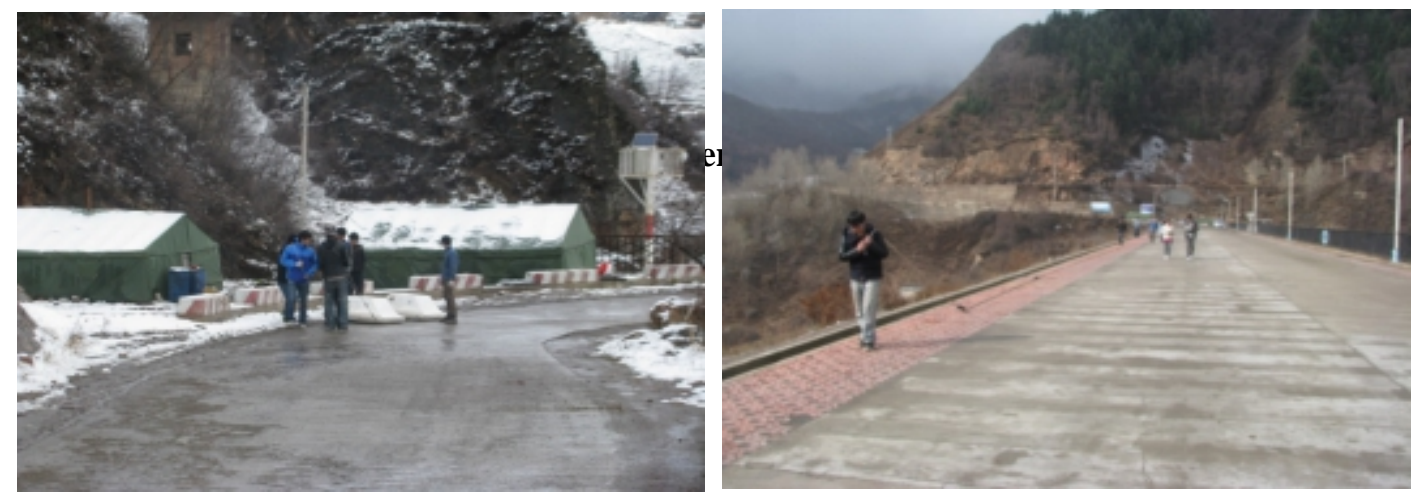

Figure 3 GV6, GV2 measured at the crest of the dam 


\section{Interpretation and validation of test data of dam foundation}

Through GV2 GPR test and analysis in the right bank of the dam grouting tunnel, the test results showed that the $0+450$ to $0+470$ m section of right bank of the grouting tunnel exists obvious fractured zones, as shown in Figure 4. it need to be paid full attention in reinforcing.

Through GV6 GPR test at bottom slope of dam that was parallel to the dam axis, found the bedrock under overburden exist significant strong penetration band in the range of $0+155$ to $0+205$, as shown in Figure 5.

Drilling holes at $0+160,0+180$ and $0+200$, fault fracture zone and partial unfilled fissure zone were seen. Self-water drilling emerged at the platform behind dam also proved that the monolith has strong permeability and a certain pressure resistance, as shown in Figure 6.

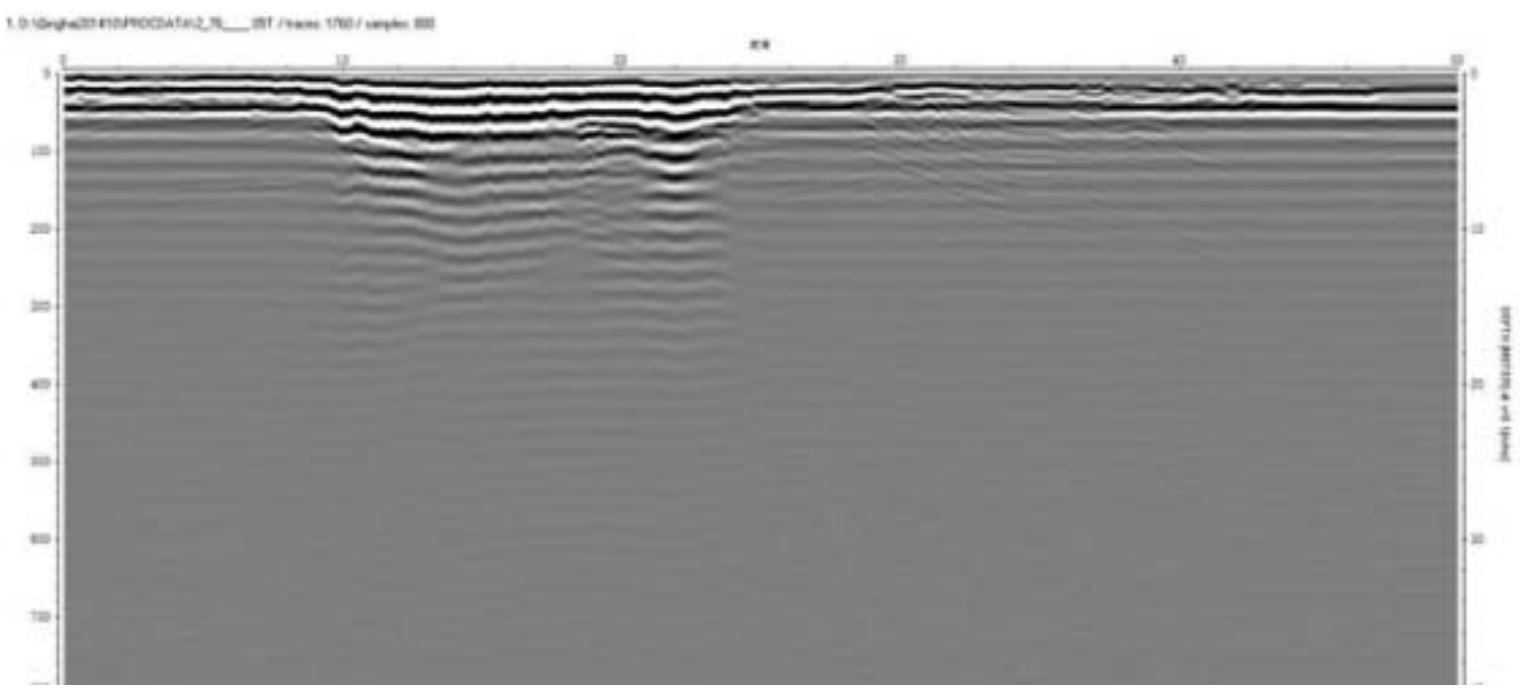

Figure 4 GV2 GPR gray scale of grouting adit on the right bank

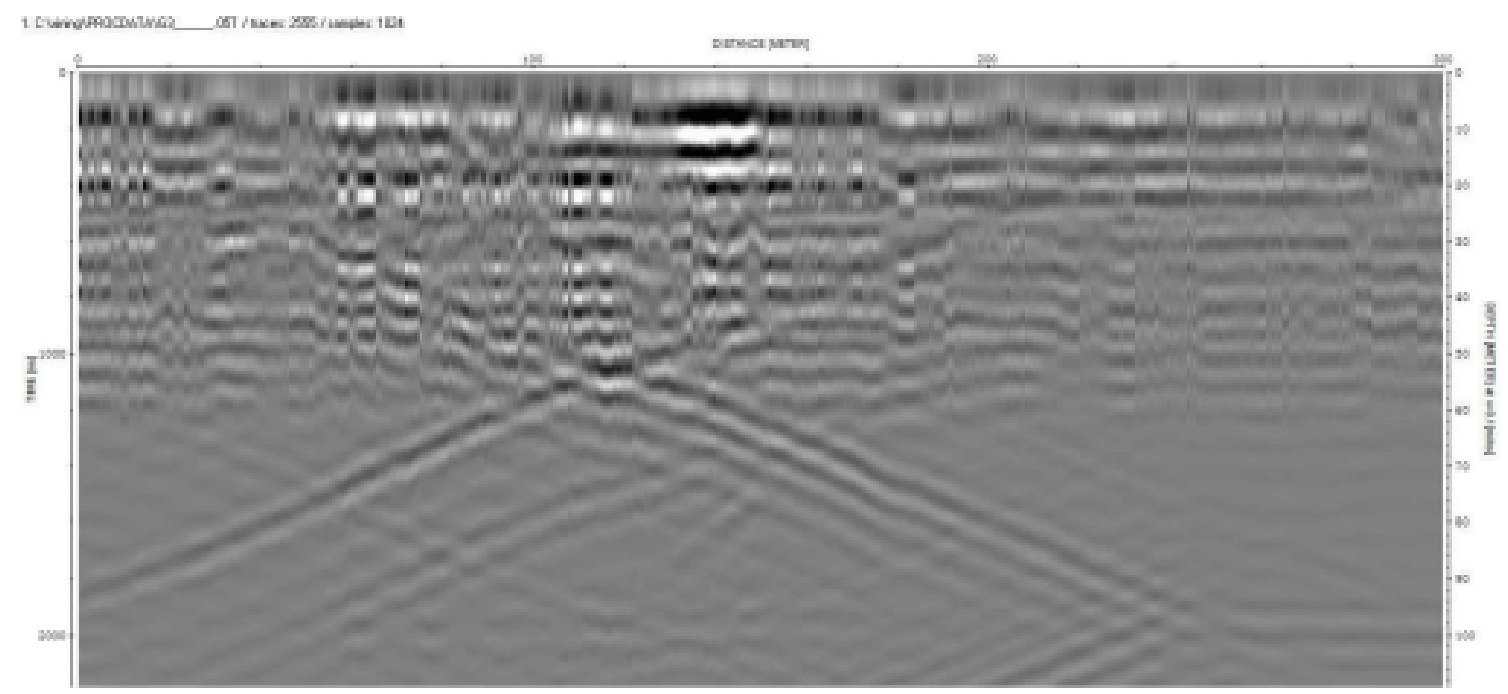

Figure5 GV6 GPR gray scale at bottom slope of dam parallel to the dam axis

\section{Conclusions}

For a long time, the seepage problem of Nanmenxia Reservoir has not been able to solve better, and the utility problem of anti-seepage wall may be a key problem, so, the important support is provided for the solution to the key problem by introducing British Groundvue geological radar equipment and software. 
Found and determined that obvious strong permeability zone existed in the dam foundation rock by GV6 test and analysis, fracture zone could be seen in drilling verification holes at $0+160,0+180$ and $0+200$. Local part has no-fill fissure zone, which was the main leakage passage of deep dam foundation.

Through test and analysis by GV2 radar, there has obvious fissure zone in local of the right grouting tunnel to be found, which should be paid full attention in reinforcement.

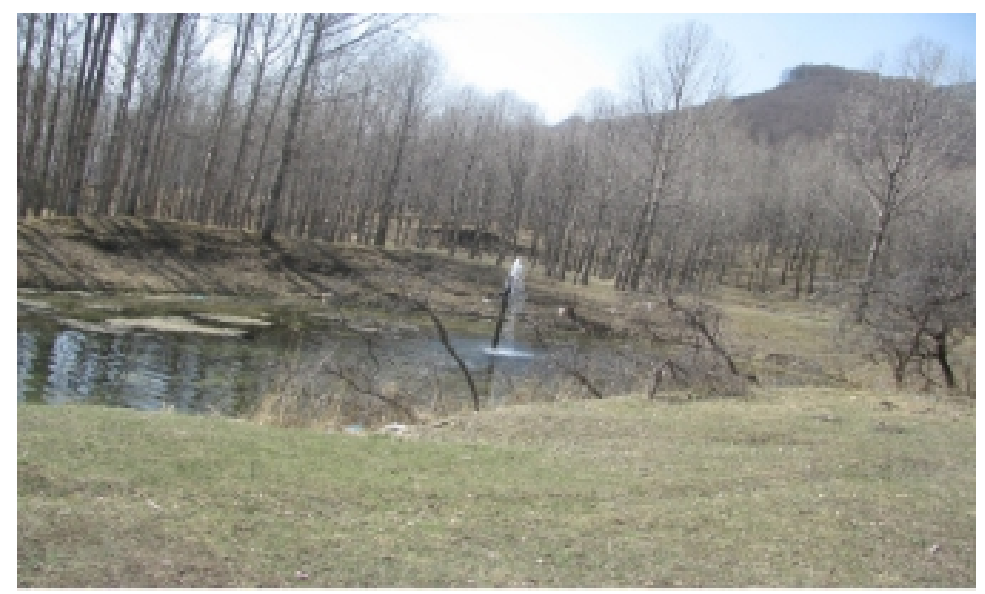

Figure 6 Self-water drilling at the platform behind dam

As the indoor test platform of GV3-1G antenna is able to effectively simulate and test the radar image characteristics of underground target objects, its results help to improve the detection interpretation efficiency of Groundvue geological radar.

\section{Acknowledgements}

This work was financially supported by the 948 Foundation of the Ministry of Water Resources (201325)

\section{References}

[1] Chen Yiqun, On the status quo and development of ground penetrating radar, J. Chinese Journal of Engineering Geophysics .16(2005)51-55.

[2] Ma Huijie. The karsts and seepage treatment of Nanmenxia Reservoir, J. Hydrogeology and Engineering Geology. 21(2002)43-48.

[3] LI Y, CHEN J J C. Ground penetrating radar signal processing improves mapping accuracy of underground voids and seawater table: an application in deteriorating coastal structure, Nanfangao Port, Taiwan, J. Enviro- nmental Geology, 43(2007)35-39.

[4] Yang Wanke, Yang Yueju, Ma Shouzhong, Reasons analysis on Nanmenxia Reservoir seepage in Huzhu. J. Science and Technology of Qinghai Agriculture and Forestry, 18(2006)43-47. 